04

\title{
Определение материальных параметров фоторефрактивных кристаллов на основе метода адаптивной голографической интерферометрии
}

\author{
(C) С.М. Шандаров ${ }^{1}$, А.О. Злобин ${ }^{1}$, А.А. Шмидт ${ }^{1}$, Н.И. Буримов ${ }^{1, \uparrow}$, К.П. Мельник ${ }^{1}$, С.С. Шмаков ${ }^{1}$, \\ А.В. Макаревич ${ }^{2}$, В.Н. Навныко ${ }^{2}$, М.А. Брюшинин ${ }^{3}$, И.А. Соколов ${ }^{3}$ \\ ${ }^{1}$ Томский государственный университет систем управления и радиоэлектроники, \\ 634050 Томск, Россия \\ ${ }^{2}$ Мозырский государственный педагогический университет им. И.П. Шамякина, \\ 247760 Мозырь, Республика Беларусь \\ ${ }^{3}$ ФТИ им. А.Ф. Иофрфе РАН, \\ 194021 Санкт-Петербург, Россия \\ ฯ e-mail: nikolai.i.burimov@tusur.ru
}

Поступила в редакцию 16.12.2020 г.

В окончательной редакции 22.12.2020 г.

Принята к публикации 22.12.2020 г.

Рассмотрено применение методов голографической интерферометрии, основанных на взаимодействии стационарного опорного светового пучка с фазово-модулированным сигнальным пучком на создаваемых ими возмущениях диэлектрического тензора в фоточувствительных кристаллах, для измерения материальных параметров, определяющих величину этих возмущений. Определены экспериментальные условия, при которых возможно выделение вкладов во взаимодействие флексоэлектрического и электрогирационного эффектов в кубических фоторефрактивных кристаллах класса силленитов; показана необходимость учета вклада в него эффекта флексогирации.

Ключевые слова: адаптивная голографическая интерферометрия, фоточувствительные кристаллы, фоторефрактивный эффект, обратный флексоэлектрический эффект, двухпучковое взаимодействие.

DOI: $10.21883 /$ OS.2021.04.50768.306-20

\section{Введение}

Еще в 1962 г. в [1] при обсуждении возможности детектирования механических колебаний объектов с предельно малыми амплитудами, вызванных гравитационными волнами, предлагалось использовать для этих целей метод лазерной интерферометрии. Предельная чувствительность к амплитуде измеряемых вибраций для этого метода, основанного на преобразовании фазовой модуляции света в модуляцию его интенсивности, ограничена дробовыми шумами фотодетектора [2], и при использовании лазера с длиной волны $633 \mathrm{~nm}$ и мощностью $1 \mathrm{~mW}$ оценивается как $10^{-15} \mathrm{~m} / \mathrm{Hz}^{1 / 2}$. Однако интерферометрические измерительные системы требуют минимизации влияния на них неконтролируемых внешних воздействий, таких как температура и механические вибрации. Другой проблемой таких систем является поддержание постоянным среднего фазового сдвига между сигнальной и опорной волнами, т.е. стабилизация рабочей точки интерферометра. Она необходима для реализации линейности амплитудной характеристики, определяющей связь сигнала, выделяемого фотодетектором, с амплитудой измеряемых колебаний. Для обеспечения адаптивности лазерных интерферометрических систем к внешним воздействиям и стабильности характеристик реализуемой ими фазовой демодуляции в [3] было предложено использование взаимодействия между сигнальным световым пучком и стационарной опорной волной на объемной динамической голограмме, формируемой ими в фоторефрактивном кристалле. Данный метод адаптивной голографической интерферометрии стал основой для многочисленных работ (см., например, [4-10]), посвященных как анализу физических явлений при взаимодействии на фоторефрактивных голограммах двух световых волн, одна из которых является фазово-модулированной, так и разработке реальных измерительных устройств и систем. Имеющие динамический характер голограммы в реверсивной среде при конечном времени ее быстродействия $\tau$ обеспечивают компенсацию дрейфа рабочей точки интерферометра для возмущений с частотами $\Omega_{e} \ll 1 / \tau$, вызванных изменением внешних условий. Двухволновое взаимодействие на динамических голограммах в фоторефрактивном кристалле, представляющем реверсивную среду, позволяет реализовать адаптивную фазовую демодуляцию сигнального пучка, несущего информацию о нестационарных возмущениях с частотами $\Omega>1 / \tau$.

Сигнал фазовой демодуляции в фоторефрактивных кристаллах характеризуется временным спектром, характер которого определяется видом отклика среды при заданных поляризационных параметрах взаимодействующих световых пучков. Соотношения между ам- 
плитудами составляющих этого спектра, регистрируемого фотоприемным устройством, зависят также от кристаллографической ориентации образца и вклада в него различных механизмов возмущения оптических свойств формируемой динамической голограммой (см., например, [4-7,10-13]). Высокая чувствительность эффекта фазовой демодуляции к возмущениям оптических свойств среды взаимодействия позволяет успешно применять методы голографической интерферометрии для определения величины вклада в нее различных вторичных эффектов и соответствующих им материальных параметров фоторефрактивных кристаллов [11-15]. Такими вторичными эффектами в кубических гиротропных кристаллах класса силленитов $\mathrm{Bi}_{12} \mathrm{SiO}_{20}, \mathrm{Bi}_{12} \mathrm{TiO}_{20}$ и $\mathrm{Bi}_{12} \mathrm{GeO}_{20}$, широко используемых при реализации устройств адаптивной голографической интерферометрии (см., например, [3-6,8-10,16-19]), являются, в частности, обратный флексоэлектрический эффект [11-13] и электрогирация [20]. Применение метода адаптивной голографической интерферометрии к встречному двухволновому взаимодействию в кристаллах $\mathrm{Bi}_{12} \mathrm{TiO}_{20}$ среза (100) позволило обнаружить в них вклад в сигнал фазовой демодуляции, обусловленный обратным флексоэлектрическим эффектом, и оценить величину компоненты $f_{1111}$ тензора коэффициентов флексоэлектрической связи [11-13]. Влияние электрогирации на эффекты энергообмена в кристаллах силленитов среза (110) при взаимодействии двух стационарных (в отсутствие фазовой модуляции) световых пучков с одинаковой циркулярной поляризацией, левой и правой, на формируемой ими фоторефрактивной решетке с вектором K $\|$ (001) рассматривалось в [21]. Отмечалось, что знак дополнительного вклада электрогирации в энергообмен связан со знаком циркулярной поляризации, и этот эффект может как усиливать, так и ослаблять основной вклад в него, определяемый линейным электрооптическим эффектом. Однако результаты экспериментальных измерений электрогирационного коэффициента $\gamma_{41}$ в кристаллах силленитов, методика которых основана на регистрации изменений поляризационных характеристик зондирующего светового пучка, прошедшего через образец с приложенным к нему внешним электрическим полем, характеризуются большим разбросом [22-30].

В настоящей работе представлены результаты теоретического анализа и экспериментального исследования фазовой демодуляции при попутном взаимодействии волн с одинаковой циркулярной поляризацией в кристалле силиката висмута среза (110). Для ориентации вектора фоторефрактивной решетки $\mathbf{K} \|(00 \overline{1})$ продемонстрирована возможность оценки из экспериментальных данных его коэффициента флексоэлектрической связи $f_{1111}$, электрогирационной постоянной $\gamma_{41}$ и эффективного флексогирационного параметра $\beta_{1122}+\beta_{2211}$.

\section{Фазовая демодуляция при попутном взаимодействии волн с циркулярной поляризацией в кристаллах класса силленитов}

Взаимодействие на фоторефрактивной голограмме сильной стационарной опорной волны со слабой сигнальной волной, несущей информацию в виде модуляции ее фазы, приводит к эффекту фазовой демодуляции $[5,31]$. При гармоническом модулирующем сигнале с частотой $\Omega$ интенсивность сигнальной волны на выходной грани кристалла может быть представлена в виде разложения

$$
\begin{aligned}
I_{S}\left(\varphi_{m}, t\right) \sim & I_{S 0}\left[M^{(0)}\left(\varphi_{m}\right)+M^{(1)}\left(\varphi_{m}\right) \sin \Omega t\right. \\
& \left.+M^{(2)}\left(\varphi_{m}\right) \cos 2 \Omega t+\ldots\right]
\end{aligned}
$$

в котором относительные амплитуды гармоник $M^{(n)}$ определяются глубиной фазовой модуляции $\varphi_{m}$, видами и параметрами векторов поляризации взаимодействующих волн на входной грани кристалла, типом фоторефрактивного отклика и его количественными характеристиками для рассматриваемого двухволнового взаимодействия. Использование рассмотренного в [11-13] подхода к анализу попутного взаимодействия световых волн с чисто циркулярной поляризацией на фоторефрактивных голограммах диффузионного типа в кристаллах класса силленитов, учитывающего вклад в нелинейный отклик обратного флексоэлектрического эффекта, приводит к следующим выражениям для относительных амплитуд первой и второй гармоник в информационном сигнале в случае образцов среза (110):

$$
\begin{aligned}
& M^{(1)}\left(\varphi_{m}\right)=4 M_{1 m} J_{0}\left(\varphi_{m}\right) J_{1}\left(\varphi_{m}\right), \\
& M^{(2)}\left(\varphi_{m}\right)=4 M_{2 m} J_{0}\left(\varphi_{m}\right) J_{2}\left(\varphi_{m}\right),
\end{aligned}
$$

где введены обозначения для эффективных параметров демодуляции на первой и второй гармониках,

$$
\begin{gathered}
M_{1 m}=\exp \left(-\frac{\Gamma_{E}}{2} d\right) \sin \left(\frac{\Gamma_{f}}{2} d\right), \\
M_{2 m}=\exp \left(-\frac{\Gamma_{E}}{2} d\right) \cos \left(\frac{\Gamma_{f}}{2} d\right)-1 .
\end{gathered}
$$

Здесь $J_{n}\left(\varphi_{m}\right)$ - функция Бесселя $n$-го порядка и $d-$ толщина кристалла, а коэффициенты связи $\Gamma_{E}$ и $\Gamma_{f}$ характеризуют вклад в попутное взаимодействие волн c одинаковой циркулярной поляризацией, связанный соответственно с линейным электрооптическим эффектом Поккельса и с обратным флексоэлектрическим эффектом. В рассматриваемом случае принималось, что эти коэффициенты малы и удовлетворяют неравенствам $\Gamma_{E}, \Gamma_{f} \ll 4 \rho$, где $\rho$ - удельное оптическое вращение кристалла. Они не зависят от знака циркулярной поляризации взаимодействующих волн и при ориентации 


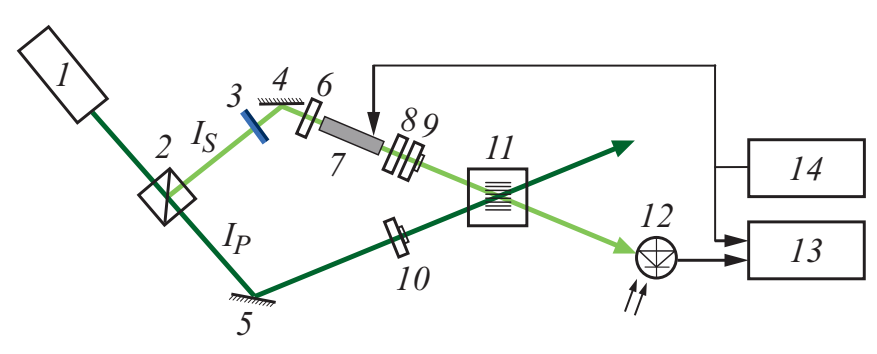

Схема экспериментальной установки для измерения материальных параметров кристаллов класса силленитов на основе метода адаптивной голографической интерферометрии при попутном двухпучковом взаимодействии. 1 - твердотельный лазер; 2 - оптический делитель; 3 - светофильтры; 4,5 зеркала; 6 - призма Глана; 7 - фазовый электрооптический модулятор; 8 - дихроичный поляризатор; 9,10 - перестраиваемые четвертьволновые пластины; 11 - фоторефрактивный кристалл $\mathrm{Bi}_{12} \mathrm{SiO}_{20}$ среза (110); 12 - фотодиод; 13 - цифровой осциллограф; 14 - генератор низкочастотных сигналов.

вектора фоторефрактивной решетки К вдоль кристаллографического направления [001] определяются выражениями

$$
\begin{gathered}
\Gamma_{E}=-\frac{2 \pi}{\lambda} \frac{n_{0}^{3} r_{41}^{S}}{2}\left(1+\sin ^{2} \theta\right) \cos \theta E_{S C} \\
\Gamma_{f}=\frac{2 \pi}{\lambda} n_{0}^{3} \frac{\left(2 p_{11}+p_{12}+p_{13}\right)}{4 c_{11}}\left(\frac{2 \pi}{\Lambda}\right) f_{1111} \cos ^{3} \theta E_{S C}
\end{gathered}
$$

где $\lambda$ - длина волны света; $n_{0}$ - показатель преломления для невозмущенного кристалла; $r_{41}^{S}-$ компонента электрооптического тензора механически зажатого кристалла; $p_{11}, p_{12}$ и $p_{13}$ - упругооптические коэффициенты кристалла; $E_{S C}$ - эффективное поле пространственного заряда голограммы $[4,11] ; c_{11}-$ компонента тензора модулей упругости кристалла в сокращенной форме записи и $\Lambda=2 \pi /|\mathbf{K}|-$ пространственный период фоторефрактивной голограммы. Угол в кристалле между волновыми векторами взаимодействующих световых волн считается здесь равным $2 \theta$, а его биссектриса направленной вдоль кристаллографической оси [110]. Отметим, что в отсутствие флексоэлектрического вклада (при $\left.\Gamma_{f}=0\right)$ линейный электрооптический эффект в рассматриваемом случае попутного взаимодействия на фоторефрактивной голограмме диффузионного типа определяет амплитуду второй гармоники выходного сигнала (см. формулы (3), (5) и (4)), используемой для измерительных систем с квадратичным режимом фазовой демодуляции [5,6]. Линейный режим демодуляции, в котором в голографическом интерферометре реализуется измерение амплитуды первой гармоники, определяется коэффициентом связи $\Gamma_{f}$, пропорциональным компоненте тензора флексоэлектрической связи исследуемого кристалла $f_{1111}$ [11-13].

\section{Эксперимент}

В экспериментах использовался монокристаллический образец $\mathrm{Bi}_{12} \mathrm{SiO}_{20}(\mathrm{BSO})$ среза (110) с просветленными гранями, имеющий толщину $d=2.2 \mathrm{~mm}$ и поперечные размеры $15 \times 15 \mathrm{~mm}^{2}$. Схема экспериментальной установки для измерения материальных параметров данного образца на основе метода адаптивной голографической интерферометрии в пропускающей геометрии представлена на рисунке.

В качестве источника излучения использовался одночастотный твердотельный лазер $(\lambda=532 \mathrm{~nm}$, мощность $5 \mathrm{~mW}$, апертура пучка $1.1 \mathrm{~mm}$ ). Делительный куб 2 разделял лазерный пучок с вектором поляризации, ортогональной плоскости рисунка, на опорный $\left(I_{p}\right)$ и сигнальный $\left(I_{s}\right)$ пучки; интенсивность сигнального пучка уменьшалась до уровня $I_{s} \approx I_{p} / 60$ набором светофильтров 3. Призма Глана 6 корректировала поляризацию света на входе электрооптического модулятора МЛ-102А для обеспечения его работы в режиме модуляции фазы. Поляризатор 8 в сочетании с четвертьволновой пластиной 9 позволял получить модулированный по фазе на частоте $\Omega / 2 \pi=1200 \mathrm{~Hz}$ сигнальный пучок с правой или левой циркулярной поляризацией. Такая же четвертьволновая пластина 10 использовалась для задания для стационарного опорного пучка соответствующее сигнальному пучку состояние циркулярной поляризации, правой или левой. Угол между каждым из световых пучков и нормалью к входной грани образца в воздухе составлял $30^{\circ}$. Интерферируя между собой, сигнальный и опорный пучки формировали в исследуемом кристалле BSO среза (110) стационарную фоторефрактивную голограмму, которая и позволяла преобразовать фазовую модуляцию входного сигнального пучка в его модуляцию по интенсивности на выходе образца. После взаимодействия модулированный по интенсивности сигнальный пучок детектируется фотоприемником 12 (фотодиод BPW-34, сопротивление нагрузки $51 \mathrm{k} \Omega$, напряжение смещения $4.9 \mathrm{~V}$ ). Электрический сигнал фазовой демодуляции, в соответствии с соотношением (1) представляющий набор временных гармоник, поступал на открытый вход цифрового осциллографа Tektronix TDS-2012C. Специализированная компьютерная система обработки данных MOHNENBLUEHEN [32] позволяла в цифровом виде фиксировать в моменты времени $t_{i}$ выборки сигнала фазовой демодуляции с длительностью около $2.5 \mathrm{~ms}$, с интервалом в $1 \mathrm{~s}$ между ними. Далее эти цифровые выборки аппроксимировались разложением на временные гармоники с частотами $n \Omega$, с определением их амплитуд для $n$ от 0 до 5 .

Образец $\mathrm{Bi}_{12} \mathrm{SiO}_{20}$ размещался в держателе, позволявшем поворачивать его в диапазоне углов от 0 до $360^{\circ}$ вокруг горизонтальной оси, совпадающей с биссектрисой угла между записывающими голограмму световыми пучками и кристаллографическим направлением [110]. Это сделало возможной регистрацию ориентационной зависимости для эффективного параметра демодуляции 
Эффективные параметры фазовой демодуляции при ориентации вектора фоторефрактивной решетки $\mathbf{K}$ в образце BSO среза (110) вдоль кристаллографического направления [001]

\begin{tabular}{c|c}
\hline Параметр & Значение \\
\hline$M_{1 m}^{r}$ & $(3.3 \pm 0.4) \cdot 10^{-3}$ \\
\hline$M_{2 m}^{r}$ & $(76.2 \pm 0.9) \cdot 10^{-3}$ \\
\hline$M_{1 m}^{l}$ & $(0.6 \pm 0.3) \cdot 10^{-3}$ \\
\hline$M_{2 m}^{l}$ & $(82.4 \pm 1.0) \cdot 10^{-3}$
\end{tabular}

по второй гармонике $M_{2 m}$ от угла $\theta_{G}$ между вектором решетки К и осью [001] кристалла. Локальные минимумы на этой зависимости $M_{2 m}\left(\theta_{G}\right)$ соответствуют ориентации $\mathbf{K} \|[001]\left(\theta_{G}=0^{\circ}\right)$ и $\mathbf{K} \|[00 \overline{1}]\left(\theta_{G}=180^{\circ}\right)$. Результаты измерения для последней ориентации образца эффективных параметров демодуляции для правой $\left(M_{1 m}^{r}, M_{2 m}^{r}\right)$ и левой $\left(M_{1 m}^{l}, M_{2 m}^{l}\right)$ циркулярной поляризации взаимодействующих пучков представлены в таблице.

Из таблицы видно, что значения эффективных параметров фазовой демодуляции зависят от знака циркулярной поляризации; при этом наблюдаемые различия превосходят экспериментальные ошибки.

\section{Обсуждение результатов}

Как следует из сравнения приведенной выше теоретической модели для обоих эффективных параметров фазовой демодуляции с экспериментальными данными (таблица), для исследованного образца BSO существуют дополнительные физические механизмы возмущения его оптических свойств электрическим полем фоторефрактивной голограммы. Такие дополнительные механизмы могут быть связаны как непосредственно с амплитудой поля пространственного заряда, так и с его градиентом. Как уже отмечалось выше, в кристаллах $\mathrm{Bi}_{12} \mathrm{SiO}_{20}$ может наблюдаться эффект электрогирации [20,24-26,28-30], заключающийся во влиянии электрического поля на удельную оптическую активность. Можно предположить, что градиент напряженности электрического поля также может возмущать удельное оптическое вращение кристалла $\rho$. Используя подход, рассмотренный в [20], представим псевдотензор гирации кристалла $g_{k l}$ в виде разложения

$$
g_{k l}=g_{0} \delta_{k l}+\gamma_{k l m} E_{m}+\beta_{k l m n} \frac{\partial E_{m}}{\partial \chi_{n}},
$$

в котором $g_{0}$ - тензор гирации в отсутствие поля, а тензор $\gamma_{k l m}$ описывает известный эффект электрогирации. Тензор четвертого ранга с компонентами $\beta_{k l m n}$, имеющий такую же симметрию, как тензор флексоэлектрической связи $f_{k l m n}$, будем называть тензором флексогирации.

Анализ показывает, что с учетом эффектов электрои флексогирации в выражениях (4) и (5) коэффициенты связи, описывающие взаимодействие волн с правой и левой циркулярными поляризациями в кристаллах класса силленитов среза (100) при ориентации вектора фоторефрактивной решетки K $\|$ [001] , должны быть представлены в виде

$$
\begin{gathered}
\Gamma_{E}^{r, l}=-\frac{2 \pi}{\lambda} n_{0}^{3}\left[\frac{r_{41}^{S}}{2}\left(1+\sin ^{2} \theta\right) \mp \gamma_{41} \cos \theta\right] \cos \theta E_{S C}, \\
\Gamma_{f}^{r, l}=\frac{2 \pi}{\lambda} n_{0}^{3}\left[\frac{\left(2 p_{11}+p_{12}+p_{13}\right)}{4 c_{11}} f_{1111} \cos \theta\right. \\
\left. \pm \frac{\beta_{1122}+\beta_{2211}}{2}\right]\left(\frac{2 \pi}{\Lambda}\right) \cos ^{2} \theta E_{S C} .
\end{gathered}
$$

Соотношения (4) и (5) и экспериментальные данные из табл. 1 позволяют найти следующие значения для коэффициентов связи: $\Gamma_{E}^{r}=-66.8 \mathrm{~m}^{-1}$, $\Gamma_{E}^{l}=-72.0 \mathrm{~m}^{-1}, \Gamma_{f}^{r}=2.80 \mathrm{~m}^{-1}$ и $\Gamma_{f}^{l}=0.53 \mathrm{~m}^{-1}$. Из них на основании формул (9) и (10), используя значения известных материальных констант кристалла $\mathrm{Bi}_{12} \mathrm{SiO}_{20}[4,14]$, получаем следующие оценки его параметров, определяющих вклад в рассматриваемое попутное взаимодействие циркулярно-поляризованных волн: $f_{1111}=9.9 \mathrm{nC} / \mathrm{m}, \quad \gamma_{41}=-0.2 \mathrm{pV} / \mathrm{m}$ и $\beta_{1122}+\beta_{2211}=-2.6 \cdot 10^{-19} \mathrm{~m}^{2} / \mathrm{V}$.

\section{Заключение}

Таким образом, результаты теоретического анализа и экспериментального исследования фазовой демодуляции при попутном взаимодействии волн с одинаковой циркулярной поляризацией в кристалле силиката висмута среза (110) показали возможность оценки его неизвестных материальных параметров, таких как коэффициент флексоэлектрической связи $f_{1111}$, электрогирационная постоянная $\gamma_{41}$ и эффективный флексогирационный параметр $\beta_{1122}+\beta_{2211}$. Высокая чувствительность метода адаптивной голографической интерферометрии к возмущениям оптических свойств среды взаимодействия позволяет успешно применять его для определения материальных параметров фоторефрактивных кристаллов.

\section{Благодарности}

Авторы выражают благодарности В.В. Шепелевичу, B.М. Петрову, В.Н. Трещикову, В.Т. Потапову и В.К. Горчакову за стимулирующие дискуссии и полезные замечания и обсуждения.

\section{Финансирование}

Работа выполнена при финансовой поддержке Министерства науки и высшего образования Российской Федерации в рамках Госзадания на 2020-2022 гг. (задание FEWM-2020-0038/3).

\section{Конфликт интересов}

Авторы заявляют, что у них нет конфликта интересов. 


\section{Список литературы}

[1] Гериенштейн М.Е., Пустовойт В.И. // ЖЭТФ. 1962. Т. 43. № 2. C. 605-607.

[2] Wagner J.W., Spicer J.B. //J. Opt. Soc. Am. B. 1987. V. 4. P. $1316-1326$.

[3] Hall T.J., Ner M.S., Fiddy M.A. // Opt. Lett. 1980. V. 5. N 11. P. $485-487$.

[4] Петров М.П., Степанов С.И., Хоменко А.В. Фоторефрактивные кристаллы в когерентной оптике. СПб.: Наука, 1992. $320 \mathrm{c}$.

[5] Колегов А.А., Шандаров С.М., Симонова Г.В., Кабанова Л.А., Буримов Н.И., Шмаков С.С., Быков В.И., Каргин Ю.Ф. // Квант. электрон. 2011. Т. 41. № 9. С. 847-852.

[6] Кульчин Ю.Н., Витрик О.Б., Камшилин А.А., Ромашко Р.B. Адаптивные методы обработки спеклмодулированных оптических полей. М.: Наука, 1973. 321 с.

[7] Ромашко Р. В., Безрук М. Н., Камшилин А. А., Кульчин Ю.Н. // Квант. электрон. 2012. Т. 42. № 6. С. 551-556.

[8] Stepanov S.I. // Rep. Prog. Phys. 1994. V. 39. P. 39-116.

[9] Solymar L., Webb D.J., Grunnet-Jepsen A. The physics and application of photorefractive materials. Oxford: Clarendon Press, 1996. 496 p.

[10] Kamshilin A.A., Romashko R.V., Kulchin Yu.N. // J. Appl. Phys. 2009. V. 105. N 3. P. 031101.

[11] Шандаров С.М., Шмаков С.С., Буримов Н.И., Сюваева О.С., Каргин Ю.Ф., Петров В.М. // Письма в ЖЭТФ. 2012. T. 95. № 12. C. 699-702.

[12] Шандаров С.М., Шмаков С.С., Зуев П.В., Буримов Н.И., Каргин Ю.Ф., Шепелевич В.В., Ропот П.И., Гуделев В.Г. // Оптический журнал. 2013. Т. 80. № 7. С. 5-12.

[13] Шандаров С.М., Буримов Н.И., Шмаков С.С., Зуев П.В., Урбан А.Е., Горбачев В.С., Каргин Ю.Ф., Шепелевич В.В. // Известия РАН. Серия физическая. 2012. Т. 76. № 12. C. $1452-1455$.

[14] Степанов С.И., Шандаров С.М., Хатьков Н.Д. // ФТТ. 1987. T. 29. № 10. С. 3054-3058.

[15] Шандаров С.М., Буримов Н.И., Быков В.И., Шепелевич B.B. // В сборнике: Голография. Наука и практика. Сборник трудов. 2015. С. 219-222.

[16] Kukhtatev N.V., Chen B.S., Venkateswarlu P., Salamo G., Klein M. // Opt. Commun. 1993. V. 104. P. 23-28.

[17] Georges M.P., Scauflaire V.S., Lemaire P.C. // Appl. Phys. B: Lasers and Optics. 2001. V. 72. P. 761-765.

[18] Petrov V., Hahn J., Petter J., Petrov M., Tschudi T. // Opt. Lett. 2005. V. 30. P. 3138-3140.

[19] Брюшинин М.А., Куликов В.В., Соколов И.A., Delaye P., Pauliat G. // ФТТ. 2014. № 56. C. 1158; Bryushinin M.A., Kulikov V.V., Sokolov I.A., Delaye P., Pauliat G. // Phys. Solid State. 2014. V. 56. N 6. P. 1206. doi 10.1134/S1063783414060092

[20] Влох О.Г. Явления пространственной дисперсии в параметрической кристаллооптике. Львов: Вища школа, 1984. $156 \mathrm{c}$.

[21] Воляр А.В., Кухтарев Н.В., Муравьев В.В., Савченко В.И., Семенец Т.И. // Письма в ЖТФ. 1989. Т. 15. № 4. С. 21-24.

[22] Miller A. // Phys. Rev. B. 1973. V. 8. P. 5902-5908.

[23] Fox A.J., Bruton T.M. // Appl. Phys. Lett. 1975. V. 27. N 6. P. $360-362$.

[24] Влох О.Г., Царик А.В. // Укр. физ. журн. 1977. Т. 22. № 6. C. 1032-1306.
[25] Куцаенко В.В., Потапов В.Т. // Письма в ЖЭТФ. 1986. T. 43. № 2. C. $108-110$.

[26] Vachss F., Hesselink L. // Opt. Commun. 1987. V. 62. N 3. P. 159-165.

[27] Wilde J.P., Hesselink L., McCabon S.W., Klein M.B., Rytz D., Wechsler B.A. // J. Appl. Phys. 1990. V. 67. P. 2245-2252.

[28] Nakagava K., Kajita N., Chen J., Minemoto T. // J. Appl. Phys. 1991. V. 69. P. 954-958.

[29] Tayag T.J., Batchmen T.E., Sluss J.J. // Appl. Opt. 1992. V. 31. N 5. P. 625-629.

[30] Deliolanis N.C., Vanidhis E.D., Vainos N.A. // Appl. Phys. B. 2006. V. 85. P. 591-596.

[31] Shandarov S.M., Kolegov A.A., Burimov N.I., Bykov V.I., Petrov V.M., Kargin Yu.F. // Phys. Wave Phenomena. 2009. V. 17. N 1. P. 39-44. 